\title{
Drug-Induced Liver Injury and Individual Cell Models
}

\author{
Andreas Benesic ${ }^{a, b} \quad$ Alexander L. Gerbes ${ }^{a}$ \\ a Liver Center Munich ${ }^{\circledR}$, Department of Medicine 2, University Hospital Grosshadern, Munich, and \\ ${ }^{\mathrm{b}}$ MetaHeps $\mathrm{GmbH}$, Martinsried, Germany
}

\section{Key Words}

Idiosyncratic drug-induced liver injury · Acute liver failure .

Cell models $\cdot$ Causality assessment $\cdot$ RUCAM $\cdot$ Biomarkers

\begin{abstract}
Drug-induced liver injury (DILI) is the most common cause of acute liver failure and accounts for the majority of regulatory actions on drugs. Furthermore, DILI is a relevant cause for project terminations in pharmaceutical development. The idiosyncratic form of DILI is especially a threat in late clinical development phases and postmarketing, respectively. Even the occurrence of only a few idiosyncratic DILI cases in late clinical development or postmarketing may suffice to terminate or withdraw an otherwise promising therapy. Despite advances in preclinical assessment of dose-dependent toxicity, idiosyncratic DILI is still a big challenge for in vitro research: it not only requires individualized models but also a huge number of tests. We have developed and investigated MetaHeps ${ }^{\circledR}$, a technology involving hepatocyte-like cells generated from peripheral monocytes without genetic modifications. These cells exhibit several hepatocyte-like characteristics and show donor-specific activities of drugmetabolizing enzymes. With MetaHeps we have performed in vitro investigations in patients with DILI suspicion. By investigating MetaHeps derived from DILI patients we could show increased in vitro susceptibility to the drugs involved
\end{abstract}

in the individual patients. MetaHeps testing could also rule out DILI and help to identify other causes of acute liver injury. Moreover, MetaHeps identified the causative agent in polymedicated patients. In conclusion, in vitro research of idiosyncratic DILI requires individual cell models which produce results comparable to the clinical situation. We suggest the MetaHeps technology as a novel tool to cope with these challenges of DILI.

(c) 2015 S. Karger AG, Basel

\section{Introduction}

Drug-induced liver injury (DILI) is the major cause for acute liver failure in the industrial world [1-3]. Additionally, it is a relevant burden for pharmaceutical development as one of the major causes for regulatory actions, market withdrawals and project terminations [4].

DILI is often classified as either dose dependent, with acetaminophen (paracetamol, APAP) as the most important example, or idiosyncratic, usually developing after a latency of several weeks to months, affecting only a few susceptibilities without clear dose dependency (table 1) [5]. Substantial progress has been achieved in developing cellular and animal models to better predict and identify drugs with the risk for dose-dependent hepatotoxicity. Yet the problem of idiosyncratic DILI is still unsolved,

\section{KARGER 125}

(c) 2015 S. Karger AG, Base

0257-2753/15/0334-0486\$39.50/0

E-Mail karger@karger.com

www.karger.com/ddi
Dr. Andreas Benesic

Leber Centrum München ${ }^{\circledR}$, Medizinische Klinik und Poliklinik 2

Klinikum der Universität München, Campus Grosshadern

Marchioninistrasse 15, DE-81377 München (Germany)

E-Mail andreas.benesic@med.uni-muenchen.de 
since individual susceptibility of the patient is the major determinant for this form of hepatotoxicity. In contrast to dose-dependent DILI, which can be discovered in preclinical development, idiosyncratic reactions are often only recognized in the late-stage clinical development of a drug, when a substantial number of patients are exposed. This review will focus on MetaHeps ${ }^{\circledR}$, a novel cell model which can reflect idiosyncratic drug toxicity in individual patients.

\section{Idiosyncratic DILI and Cell Models}

Metabolism and detoxification processes in the hepatocyte play an important role in the occurrence of drug toxicity [5]. Since there are substantial metabolism differences between human and animal livers, primary human hepatocytes $(\mathrm{PHH})$ are considered to be the gold standard for in vitro hepatotoxicity testing [6]. Yet the use of PHH is limited by their lack of long-term stability in culture and the limited availability of cells, as well as a very limited number of donors. Progress has been made by improving stability through improved culture conditions, taking advantage of sandwich cultures, cocultures with nonparenchymal cells and 3D microtissues. In order to overcome the limited availability of cells, tumor cell lines or immortalized hepatocytes are an option [7]. Yet these cell sources allow no (tumor cells) or limited (immortalized hepatocytes) individualization. Stem cell technologies, especially induced pluripotent stem cells may be promising alternatives (table 2) [8]. However, to date no data are available showing induced pluripotent stem cells reflecting individual susceptibility to drug hepatotoxicity. Furthermore, all these models are applied in the preclinical drug development process. Thus, in the view of the rare incidence of idiosyncratic DILI, huge numbers of tests would be required to exclude the risk of idiosyncratic DILI. Therefore, we have developed MetaHeps, a cell model that is generated from peripheral monocytes without genetic modifications [9]. Our data provide evidence that MetaHeps show several characteristics of hepatocytes necessary for the investigation of drug hepatotoxicity in vitro. Moreover, they reflect individual characteristics of the donor's hepatocytes. The cells can be cryoconserved and therefore enable the setup of a cellbank and a database. Thus far, MetaHeps have been generated and cryopreserved from more than 100 donors, including $30 \mathrm{pa}-$ tients diagnosed with idiosyncratic DILI. Almost 100 compounds with different levels of DILI concern have been tested, resulting in an extensive database.

\section{The Need for a Test to Positively Diagnose and Exclude Idiosyncratic DILI}

Idiosyncratic DILI is one of the most challenging diagnoses in hepatology which relies on accurate history, examinations and exclusion of other possible etiologies for liver injury [10]. The current gold standard for the diagnosis is expert opinion, sometimes accompanied by standardized causality assessment methods such as the CIOMS/RUCAM score [11]. Even if DILI can be established as the most likely diagnosis, polypharmacy can preclude the identification of the causative drug. On the other hand, there is also evidence that liver injuries of other etiology are often misdiagnosed as DILI [12].

An important feature in assessing causality of a drug is the typical signature, consisting, for example, of a typical time to onset or pattern of injury. Such a signature can only be described once DILI has happened and, therefore, is not available before late-stage development or the marketing of novel drugs. Diagnostic uncertainty, in combination with the risk of fatal liver injury, requires careful evaluation when increased frequencies of liver enzyme elevations are observed during clinical trials. Until now, no reliable laboratory test to assess causality of the respective drug is available [13]. Thus, the actual DILI risk might be overestimated and promising drugs are potentially being discarded due to safety concerns. Currently, there is no solution to the challenge that DILI poses on drug developers and regulators: combining maximum patient safety with efficient drug development processes, avoiding unnecessary losses of drugs and investments [14, 15].

Current risk minimization strategies rely on patterns and frequencies of liver enzyme elevations. For example, the occurrence of only one or two 'Hy's law' cases [alanine aminotransferase (ALT) elevation $>3 \mathrm{ULN}$ and bilirubin $>2 \mathrm{ULN}]$ in combination with a higher incidence of lowgrade elevations in the treatment populations in comparison to a control group might lead to discontinuation of a drug [16]. The great impact of even a single suspected case of severe DILI highlights the false importance of false-positive DILI diagnosis. False-positive results increase the risk of innovative drugs to be terminated, depriving patients of effective new treatments and causing investment losses. Thus, a diagnostic test will not only have to provide high sensitivity, excellent specificity is even more important in cases of rare events such as DILI $[17,18]$. Therefore, there is urgent need for technologies which provide objective data to diagnose or exclude DILI in individual patients and also allow the assessment of causality of a drug, even in polymedicated patients. 
Fig. 1. Spiderweb graph of the results obtained by testing drug toxicity in MetaHeps of a DILI patient. A toxicity of $>90 \%$ was observed by incubation with the drug $\mathrm{O}$ that was clinically suspected to have caused DILI in this patient. When tested in MetaHeps of healthy donors, $\mathrm{O}(10 \mu \mathrm{M})$ did not cause any toxicity (data not shown).

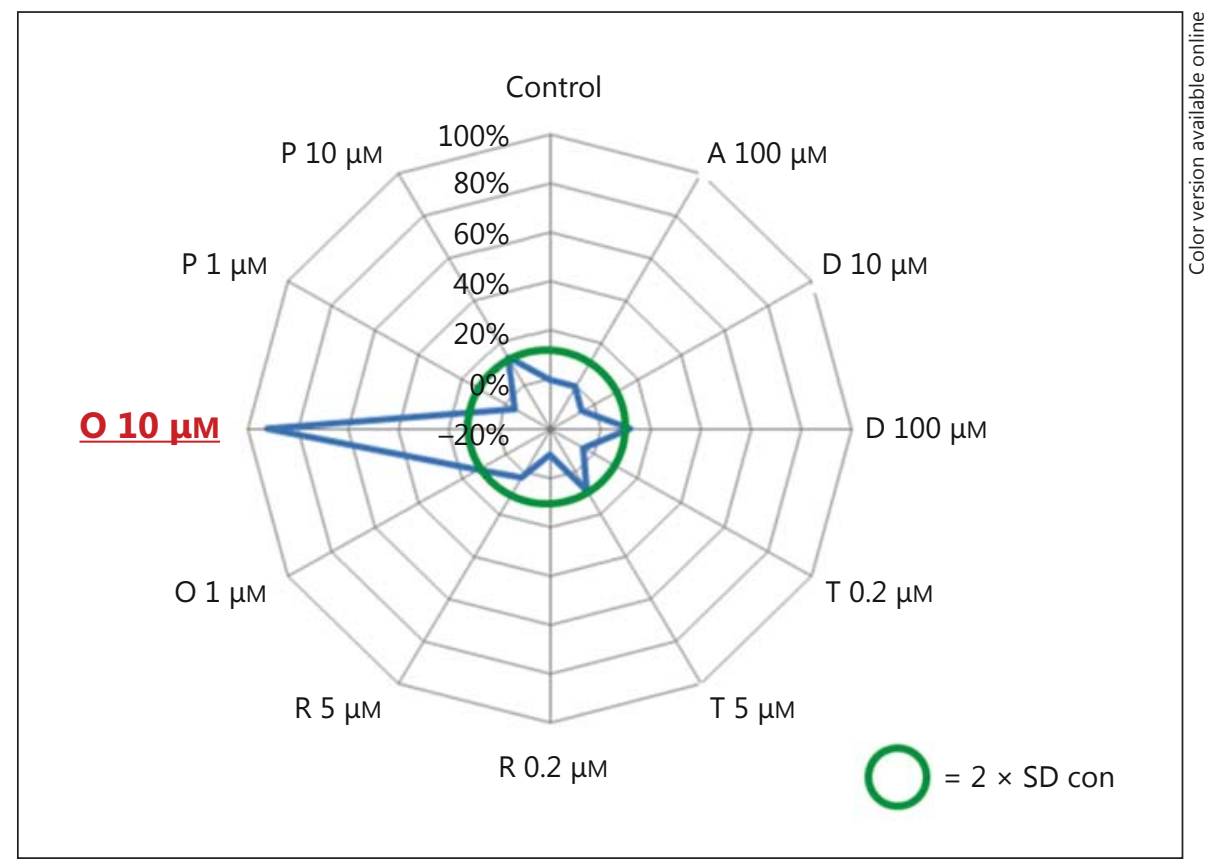

\section{MetaHeps as a Tool in the Diagnosis of Idiosyncratic DILI}

Since MetaHeps reflect individual hepatocyte characteristics of the donor, their performance as a test for idiosyncratic DILI is being investigated in our ongoing study in idiosyncratic DILI patients. The control group is represented by patients with acute liver injury and the intake of at least one drug. Additional inclusion criteria are defined according to Aithal et al. [12] as: ALT $\geq 5 \mathrm{ULN}$, alkaline phosphatase (AP) $\geq 2 \mathrm{ULN}$ or ALT $\geq 3 \mathrm{ULN}$ accompanied by a bilirubin elevation $\geq 2$ ULN. For the diagnosis or exclusion of DILI, the patient's history is obtained as well as clinical information, as proposed by Agarwal et al. [19]. Routine laboratory investigations include ALT, aspartate aminotransferase (AST), AP, $\gamma$-glutamyltransferase (GGT), bilirubin, international normalized ratio and blood cell counts. Virological testing for hepatitis A, B, C and E, as well as for HIV, cytomegalovirus and EpsteinBarr virus is performed and extended when the history provided evidence. Autoimmune hepatitis is diagnosed or excluded using ANA, IgG and, where indicated, liver biopsy. Each patient is examined by ultrasonography to exclude cholestasis; CT scan and IMR (liver MR and MRCP) are performed when additional imaging is deemed necessary. The RUCAM score is calculated for each patient and information on drug hepatotoxicity signatures is obtained using the LiverTox website [20].
Table 1. Comparison of dose-dependent and idiosyncratic DILI

Intrinsic (dose-dependent) Idiosyncratic DILI

DILI

Affects all individuals with exposure exceeding critical Affects only susceptible individuals at low frequency (e.g. 1 in 10,000)

dose

No obvious dose dependency

\begin{tabular}{ll}
\hline Clearly dose-related & No obvious dose dependency \\
\hline Short latency (days) & $\begin{array}{l}\text { Variable, but usually long latency: } \\
\text { weeks, months or even years }\end{array}$ \\
\hline Distinctive liver lesion & $\begin{array}{l}\text { Can mimic nearly every known } \\
\text { liver disease }\end{array}$ \\
\hline $\begin{array}{l}\text { Predictable in vitro and in } \\
\text { animals }\end{array}$ & $\begin{array}{l}\text { Usually not predicted by routine } \\
\text { in vitro and animal testing }\end{array}$ \\
\hline
\end{tabular}

After informed consent is obtained, MetaHeps are generated and tested with the drugs that are implicated in the respective case. Toxicity is determined by the measurement of lactate dehydrogenase in supernatant and cell lysate. Since the method allows the testing of several drugs using cells of the same donor, in vitro testing also includes drugs that were known to be tolerated by the patient. A typical result obtained with MetaHeps from a DILI patient is shown in figure 1 . 
Fig. 2. Spiderweb graph of the results obtained by testing drug toxicity in MetaHeps. This patient experienced DILI during treatment with drugs X and Y. RUCAM and available data on drug signatures could not help to identify the causative drug. In MetaHeps of this patient, drug X caused toxicity in clinically relevant concentrations, whereas drug $\mathrm{Y}$ showed no effect (both drugs showed no toxicity in the used concentrations in MetaHeps from healthy donors; data not shown). The combination of $\mathrm{X}$ and $\mathrm{Y}$ yielded the same results as drug $\mathrm{X}$ alone. Therefore, $\mathrm{X}$ seems to be responsible for DILI in this patient, whereas $\mathrm{Y}$ is cleared and there is no evidence for a relevant interaction of $\mathrm{X}$ and $\mathrm{Y}$.

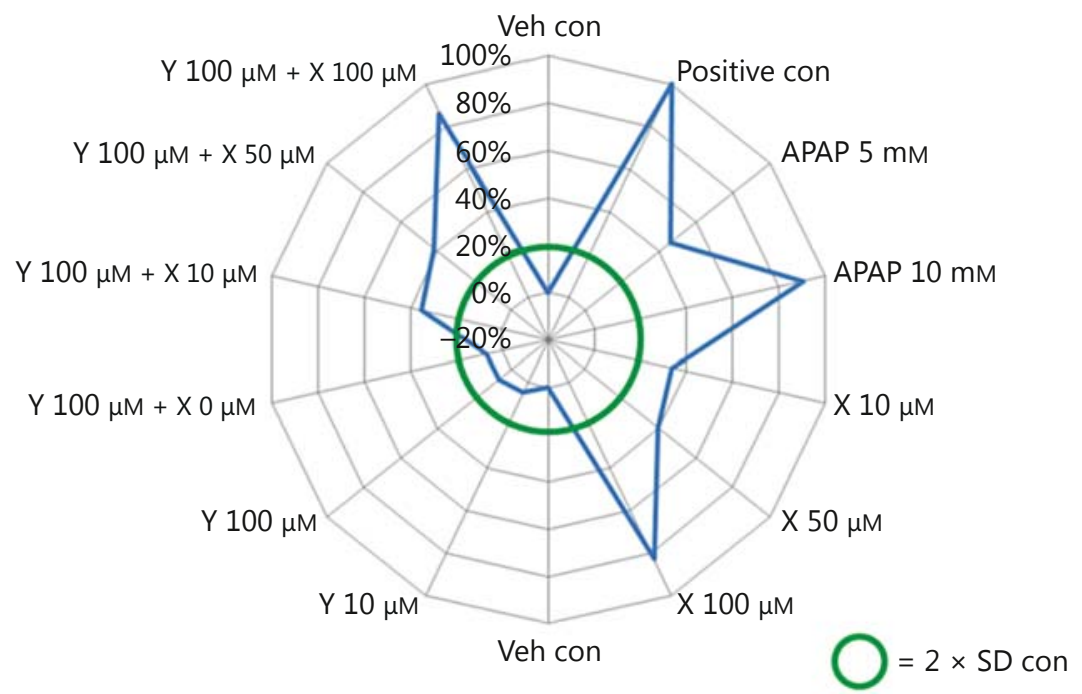

Table 2. Comparison of cell models for DILI

\begin{tabular}{|c|c|c|c|c|c|}
\hline & $\begin{array}{l}\text { Hepatocyte } \\
\text { function }\end{array}$ & $\begin{array}{l}\text { Long-term } \\
\text { stability }\end{array}$ & $\begin{array}{l}\text { Availability } \\
\text { of cells }\end{array}$ & $\begin{array}{l}\text { Availability } \\
\text { of donors }\end{array}$ & $\begin{array}{l}\text { Reflection of individual } \\
\text { hepatotoxicity }\end{array}$ \\
\hline \multicolumn{6}{|l|}{$\mathrm{PHH}$} \\
\hline $3 \mathrm{D}$ & ++ & ++ & - & + & no data \\
\hline Immortalized & + & - & + & + & no data \\
\hline \multicolumn{6}{|c|}{ Tumor cell lines (examples) } \\
\hline Huh-7 & + & - & ++ & - & - \\
\hline HepaRG & + & ++ & ++ & - & - \\
\hline \multicolumn{6}{|l|}{ Stem cells } \\
\hline Embryonic & + & + & + & - & - \\
\hline Induced pluripotent & + & + & + & + & no data \\
\hline MetaHeps & + & ++ & + & ++ & ++ \\
\hline
\end{tabular}

So far, in our 50 patients there has been no false-positive result and only 2 DILI patients were missed by MetaHeps. In the case of patients with polymedication, MetaHeps could help to identify the causative agent (an example is shown in fig. 2) [29]. These promising results warrant further validation in a larger patient sample. MetaHeps were also able to reproducibly reflect individual drug sensitivity even months after the DILI event.

DILI and Individual Cell Models

\section{Impact of Positive Identification of DILI Patients and the Causative Drug on Biomarker Development}

Due to the low frequency of hepatotoxicity observed even with withdrawn drugs (e.g. with troglitazone only $1.9 \%$ of patients developed liver enzyme elevations [17, 21]), identification of susceptibility factors has been proposed. Such predictive factors should solve the dilemma of drug developers and regulators achieving both patient 
safety and successful marketing of novel and innovative drugs. Among individual factors such as age, gender, alcohol consumption and comedication, etc., genetic risk factors have also been identified [22]. Most interestingly, genetic polymorphisms in hepatic metabolism and transport are very variably linked to the DILI risk, whereas the closest association was shown for flucloxacillin and the human leukocyte antigen HLA-B*5701 with an OR of 80.6 [23]. These findings suggest an important role for immunological mechanisms. In order to identify a safety biomarker a similar approach was used to rescue lumiracoxib, a COX-2 inhibitor, that caused liver enzyme elevations in only $3 \%$ of treated patients [24] and was implicated in several cases of severe liver injury. HLA (human leukocyte antigen) polymorphisms (the strongest association was with HLA-DRB1*1501) could be identified by a genomewide association study, yielding a positive predictive value of only 0.08 [25]. For this study, patients were selected by ALT and/or AST $\geq 3$ ULN under lumiracoxib and compared to a lumiracoxib-treated control group without liver enzyme elevations. The clinical relevance of such findings may be limited since, in contrast to the flucloxacillin study, most patients exhibiting this polymorphism were asymptomatic [26]. Thus, differentiation may be difficult between frequent asymptomatic elevations of liver enzymes and rare but severe DILI. In order to develop more effective biomarkers, unequivocal diagnosis of DILI and precise identification of the DILI-causing agent are pivotal, yet even in thoroughly performed studies it may not be possible to verify the diagnosis in up to $25 \%$ of cases [27]. Therefore, a test that allows the diagnosis or exclusion of DILI and identification of the causative agent may prove very valuable for the development of efficient biomarkers, especially for novel drugs where causality assessment is limited by lack of a known drug 'signature' [28].

\section{Conclusion and Perspectives}

Idiosyncratic DILI is still a diagnosis of exclusion and consensus expert opinion following a thorough evaluation is the current gold standard for establishing causality in patients with suspected DILI. In combination with causality assessment scores such as RUCAM the diagnosis can be ascertained in many cases, yet polymedication may make it impossible to identify a single causative agent or to clear a drug from the DILI suspicion. These remaining uncertainties require novel methods that enable the positive diagnosis of DILI and, moreover, identify the drug causing DILI in the individual patient. Our investigations with MetaHeps have shown that hepatocyte-like cells derived from peripheral monocytes provide promising data. We suggest that this approach should be further evaluated.

\section{Acknowledgements}

This work was funded by EXIST Technology Transfer (Grant No. 03EFT9BY56), German Federal Ministry of Economy and Technology, and the $\mathrm{m} 4$ Award for personalized medicine by the Bavarian State Ministry for Economic Affairs and Media, Energy and Technology.

\section{Disclosure Statement}

The authors disclose potential financial conflicts, since patent applications have been filed for the generation of $\mathrm{MH}$ cells as well as for possible applications. There are no professional nor personal conflicts to disclose.

\section{References}

1 Ostapowicz G, Fontana RJ, Schiødt FV, Larson A, Davern TJ, Han SH, McCashland TM, Shakil AO, Hay JE, Hynan L, Crippin JS, Blei AT, Samuel G, Reisch J, Lee WM: Results of a prospective study of acute liver failure at 17 tertiary care centers in the United States. Ann Intern Med 2002;137:947954.

2 Reuben A, Koch DG, Lee WM: Drug-induced acute liver failure: results of a US multicenter, prospective study. Hepatology 2010;52:20652076.
3 Larrey D, Pageaux GP: Drug-induced acute liver failure. Eur J Gastroenterol Hepatol 2005; 17:141-143.

4 Stevens JL, Baker TK: The future of drug safety testing: expanding the view and narrowing the focus. Drug Discov Today 2009;14:162167.

5 Yuan L, Kaplowitz N: Mechanisms of druginduced liver injury. Clin Liver Dis 2013;17: 507-518.

6 LeCluyse EL, Alexandre E, Hamilton GA, Viollon-Abadie C, Coon DJ, Jolley S, Richert L: Isolation and culture of primary hepatocytes. Methods Mol Biol 2005;290:207-229.
7 Gómez-Lechón MJ, Lahoz A, Gombau L, Castell JV, Donato MT: In vitro evaluation of potential hepatotoxicity induced by drugs. Curr Pharm Des 2010;16:1963-1977.

8 Anson BD, Kolaya K, Kamp TJ: Opportunities for human iPS cells in predictive toxicology. Clin Pharmacol Ther 2011;89:754-758.

9 Benesic A, Rahm NL, Ernst S, Gerbes AL: Human monocyte-derived cells with individual hepatocyte characteristics: a novel tool for personalized in vitro studies. Lab Invest 2012; 92:926-936. 
10 Verma S, Kaplowitz N: Diagnosis, management and prevention of drug-induced liver injury. Gut 2009;58:1555-1564.

11 Chalasani NP, Hayashi PH, Bonkovsky HL, Navarro VJ, Lee WM, Fontana RJ: ACG clinical guideline: the diagnosis and management of idiosyncratic drug-induced liver Injury. Am J Gastroenterol 2014;109:950-966.

12 Aithal GP, Watkins PB, Andrade RJ, Larrey D, Molokhia M, Takikawa H, Hunt CM, Wilke RA, Avigan M, Kaplowitz N, Bjornsson E, Daly AK: Case definition and phenotype standardization in drug-induced liver injury. Clin Pharmacol Ther 2011;89:806-815.

13 Berg PA, Becker EW: The lymphocyte transformation test - a debated method for the evaluation of drug allergic hepatic injury. J Hepatol 1995;22:115-118.

14 Senior JR: Drug hepatotoxicity from a regulatory perspective. Clin Liver Dis 2007;11:507524.

15 Senior JR: Evolution of the food and drug administration approach to liver safety assessment for new drugs: current status and challenges. Drug Saf 2014;37(suppl 1):9-17.

16 Lee WM, Senior JR: Recognizing drug-induced liver injury: current problems, possible solutions. Toxicol Pathol 2005;33:155-164.

17 Watkins PB: Idiosyncratic liver injury: challenges and approaches. Toxicol Pathol 2005; $33: 1-5$
18 Shi Q, Hong H, Senior J, Tong W: Biomarkers for drug-induced liver injury. Expert Rev Gastroenterol Hepatol 2010;4:225-234.

19 Agarwal AK, McHutchison JG, Hoofnagle $\mathrm{JH}$ : Important elements for the diagnosis of drug-induced liver injury. Clin Gastroenterol Hepatol 2010;8:463-470.

20 Hoofnagle JH, Serrano J, Knoben JE, Navarro VJ: LiverTox: a website on drug-induced liver injury. Hepatology 2013;57:873-874.

21 Lewis JH: 'Hy's Law', the 'Rezulin Rule', and other predictors of severe drug-induced hepatotoxicity: putting risk-benefit into perspective. Pharmacoepidemiol Drug Saf 2006;15: 221-229.

22 Chalasani N, Björnsson E: Risk factors for idiosyncratic drug-induced liver injury. Gastroenterology 2010;138:2246-2259.

23 Daly AK, Donaldson PT, Bhatnagar P, Shen Y, Pe'er I, Floratos A, Daly MJ, Goldstein DB, John S, Nelson MR, Graham J, Park BK, Dillon JF, Bernal W, Cordell HJ, Pirmohamed M, Aithal GP, Day CP: HLA-B*5701 genotype is a major determinant of drug-induced liver injury due to flucloxacillin. Nat Genet 2009;41: 816-819.
24 Schnitzer TJ, Burmester GR, Mysler E, Hochberg MC, Doherty M, Ehrsam E, Gitton X, Krammer G, Mellein B, Matchaba P, Gimona A, Hawkey CJ: Comparison of lumiracoxib with naproxen and ibuprofen in the Therapeutic Arthritis Research and Gastrointestinal Event Trial (TARGET), reduction in ulcer complications: randomised controlled trial. Lancet 2004;364:665-674.

25 Singer JB, Lewitzky S, Leroy E, Yang F, Zhao X, Klickstein L, Wright TM, Meyer J, Paulding CA: A genome-wide study identifies HLA alleles associated with lumiracoxib-related liver injury. Nat Genet 2010;42:711-714.

26 Chitturi S, Farrell GC: Identifying who is at risk of drug-induced liver injury: is human leukocyte antigen specificity the key? Hepatology 2011;53:358-362.

27 Teschke R, Frenzel C, Wolff A, Eickhoff A, Schulze J: Drug induced liver injury: accuracy of diagnosis in published reports. Ann Hepatol 2014; 13:248-255.

28 Watkins PB, Seeff LB: Drug-induced liver injury: summary of a single topic clinical research conference. Hepatology 2006;43:618631

29 Benesic A, Leitl A, Gerbes AL: Monocytederived hepatocyte-like cells for causality assessment of idiosyncratic drug-induced liver injury. Gut 2015, DOI: 10.1136/gutjnl-2015309528. 